\title{
Acoustic microstreaming produced by nonspherical oscillations of a gas bubble. I. Case of modes 0 and $\boldsymbol{m}$
}

\author{
Alexander A. Doinikov, ${ }^{1}$ Sarah Cleve, ${ }^{1}$ Gabriel Regnault, ${ }^{1}$ Cyril Mauger, ${ }^{1}$ and Claude Inserra ${ }^{2, *}$ \\ ${ }^{1}$ Univ Lyon, INSA Lyon, Ecole Centrale de Lyon, Université Claude Bernard Lyon I, CNRS, LMFA, UMR 5509, \\ F-69621 Villeurbanne, France \\ ${ }^{2}$ Univ Lyon, Université Lyon 1, Centre Léon Bérard, INSERM, LabTAU, F-69003 Lyon, France
}

(Received 19 January 2019; revised manuscript received 23 August 2019; published 10 September 2019)

\begin{abstract}
A theory is developed that allows one to model the velocity field of acoustic microstreaming produced by nonspherical oscillations of an acoustically driven gas bubble. It is assumed that some of the bubble oscillation modes are excited parametrically and hence can oscillate at frequencies different from the driving frequency. Analytical solutions are derived in terms of complex amplitudes of oscillation modes, which means that the mode amplitudes are assumed to be known and serve as input data when the velocity field of acoustic microstreaming is calculated. No restrictions are imposed on the ratio of the bubble radius to the viscous penetration depth. The present paper is the first part of our study in which a general theory is developed and then applied to the case that acoustic microstreaming is generated by the interaction of the breathing mode (mode 0 ) with a mode of arbitrary order $m \geqslant 1$. Examples of numerical simulations and a comparison with experimental results are provided.
\end{abstract}

DOI: 10.1103/PhysRevE.100.033104

\section{INTRODUCTION}

There are a number of technological, chemical, and biomedical applications in which steady vortex flows generated in a liquid by the oscillations of acoustically driven bubbles play an important role [1]. These applications inspire a longstanding research interest in the above physical phenomenon commonly known as acoustic microstreaming [2].

First experimental observations of acoustic microstreaming induced by gas bubbles were reported by Kolb and Nyborg [3] and Elder [4]. Early theoretical studies of this phenomenon are devoted to microstreaming that is produced by the dipole (translation) and monopole (pulsation) modes as in the cases of a translating bubble [5] and a bubble both translating and pulsating [6,7]. In more recent studies, attempts have been made to consider shape modes of higher order [8-10]. Maksimov [8] derived an asymptotic solution for acoustic microstreaming generated by a parametrically excited shape mode of order $n \gg 1$, assuming that the amplitude of the shape mode is much greater than that of the breathing mode so the contribution of the former to the microstreaming is dominant. This situation is observed when a millimeter-sized bubble is driven at a frequency of a few kilohertz [11]. However, Maksimov's solution is not valid for microfluidic and biomedical applications where one has to deal with micronsized bubbles driven at high $\mathrm{kHz}$ and $\mathrm{MHz}$ frequencies $[12,13]$. Doinikov and Bouakaz [9] and Spelman and Lauga [10] developed theories that include modes of all orders, but their calculations assume that all the modes oscillate at the same frequency, which means that the parametric generation of shape modes is ignored. In addition, their theories do not

*Corresponding author: claude.inserra@inserm.fr allow one to separate contributions of different modes. This makes it difficult to apply their theories in order to predict microstreaming in real experimental conditions, such as those reported by Guédra et al. [14-16], where predominant oscillatory modes are generated parametrically and hence oscillate at half driving frequency. It should also be emphasized that all of the above-mentioned studies, except for Ref. [9], assume that the bubble radius is much greater than the viscous penetration depth and therefore calculations are performed approximately, only up to leading terms in the framework of the above-stated assumption on viscous effects.

The purpose of our study is to develop a theory that allows one to model the velocity field of acoustic microstreaming produced by nonspherical oscillations of a gas bubble, assuming that some of the oscillation modes are excited parametrically and oscillate at frequencies different from the driving frequency at which the breathing mode is excited. Our derivation provides analytical solutions in terms of complex amplitudes of oscillation modes. This means that the modal amplitudes are assumed to be known (for example, because they are measured experimentally as in Refs. [14-16]) and therefore they serve as input data when the velocity field of acoustic microstreaming is calculated. An important feature of our derivation is that no restrictions are imposed on the ratio of the bubble radius to the viscous penetration depth. The complexity of our derivation and the bulkiness of equations make us divide the description of our results into two parts. The present paper is the first part of our study, in which a general theory is developed and then applied to the case that acoustic microstreaming is generated by the interaction of the breathing mode (mode 0 ) with a mode of arbitrary order $m \geqslant 1$. The second part of our study is presented in the next paper, which provides solutions for the case of acoustic microstreaming induced by the translational mode (mode 1) 

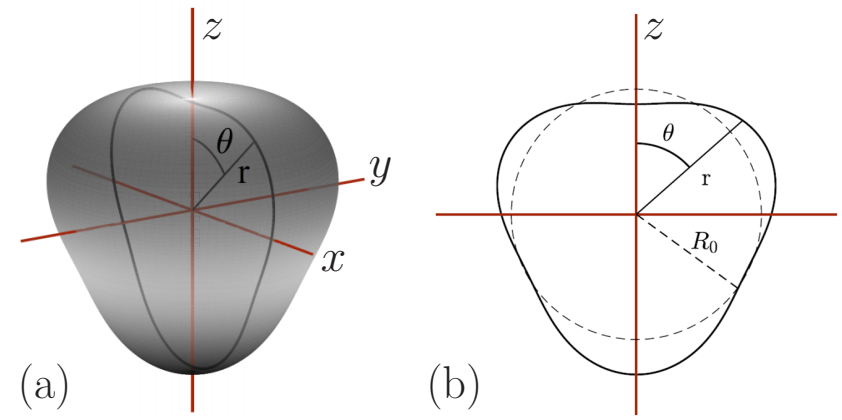

FIG. 1. Geometry of the system under study. (a) Threedimensional representation of the zonal spherical harmonics (here mode 3 is shown), where $z$ is the axis of axial symmetry. (b) Axial symmetry allows using polar coordinates $(r, \theta)$ to characterize the bubble interface.

alone and by the interaction of the translational mode with a mode of arbitrary order $m \geqslant 2$. Both parts provide examples of numerical simulations and a comparison with experimental results.

\section{THEORY}

The geometry of the system under study is depicted in Fig. 1. We assume that a single gas bubble, suspended in an infinite liquid, undergoes axisymmetric oscillations in response to an imposed acoustic field. The axis $z$ is the axis of symmetry. The liquid motion is described by the spherical coordinates $r$ and $\theta$ whose origin is at the equilibrium center of the bubble. Our derivation follows the conventional procedure. We assume that the amplitudes of the bubble oscillation modes are small compared to the equilibrium bubble radius. This assumption allows us to linearize the equations of liquid motion (Navier-Stokes equations) and to find their solutions, assuming that the amplitudes of the bubble oscillation modes are given quantities. These solutions give us a linear time-dependent velocity field produced by the bubble in the liquid. In the next step, the equations of liquid motion are written with accuracy up to terms of the second order of smallness with respect to the linear solutions and averaged over time. This operation leads to equations that describe the time-independent velocity field of acoustic microstreaming produced by the bubble oscillations. Solutions to the above equations are the ultimate aim of our derivation.

\section{A. Linear solutions}

We assume that the bubble oscillation may be decomposed into $N$ axisymmetric oscillation modes including the radial pulsation (mode 0), translation (mode 1), and shape modes (modes of order $n>1$ ). Due to the parametric behavior of nonspherical bubble dynamics, some of the modes can oscillate at frequencies different from the driving frequency. Therefore, in the general case, the bubble surface can be represented as

$$
r_{s}=R_{0}+\sum_{n=0}^{N} s_{n} e^{-i \omega_{n} t} P_{n}(\mu),
$$

where $R_{0}$ is the bubble radius at rest, $s_{n}$ is the complex amplitude of the $n$th mode, $\omega_{n}$ is the angular frequency of the $n$th mode, $\mu=\cos \theta$, and $P_{n}$ is the Legendre polynomial of order $n$. Equation (1) results from the fact that an arbitrary axisymmetric surface can be expanded in Legendre polynomials $[10,17]$.

It is assumed that $\left|s_{n}\right| / R_{0} \ll 1$. The values of $s_{n}$ and $\omega_{n}$ are considered as known quantities. They are measured experimentally and serve as input data in our study. Note also that in the general case, $\omega_{n}$ is different from the resonant frequency of the monopole bubble pulsation.

Considering that the bubble radius is small compared to the sound wavelength, the liquid compressibility can be neglected. The linearized equations of an incompressible viscous liquid are given by [18]

$$
\begin{gathered}
\boldsymbol{\nabla} \cdot \boldsymbol{v}_{1}=0 \\
\frac{\partial \boldsymbol{v}_{1}}{\partial t}=-\frac{1}{\rho} \nabla p_{1}+v \Delta \boldsymbol{v}_{1}
\end{gathered}
$$

where $\boldsymbol{v}_{1}$ and $p_{1}$ are the first-order liquid velocity and pressure, respectively, $\rho$ is the constant liquid density, $v=\eta / \rho$ is the kinematic liquid viscosity, and $\eta$ is the dynamic liquid viscosity.

A solution for $\boldsymbol{v}_{1}$ is sought as

$$
\boldsymbol{v}_{1}=\nabla \varphi_{1}+\nabla \times \boldsymbol{\psi}_{1}
$$

where $\varphi_{1}$ and $\psi_{1}$ are the scalar and the vector velocity potentials, respectively.

In view of axial symmetry, the liquid velocity has only $r$ and $\theta$ components. This fact allows us to take expressions for $\varphi_{1}$ and $\psi_{1}$ in the following form:

$$
\begin{gathered}
\varphi_{1}=\varphi_{1}(r, \theta, t), \\
\boldsymbol{\psi}_{1}=\psi_{1}(r, \theta, t) \boldsymbol{e}_{\varepsilon},
\end{gathered}
$$

where $\boldsymbol{e}_{\varepsilon}$ is the unit azimuth vector.

Substitution of Eq. (4) into Eq. (2) results in

$$
\Delta \varphi_{1}=0
$$

where $\Delta$ denotes the Laplace operator. A solution to Eq. (7), in view of Eq. (1), is given by

$$
\varphi_{1}=\sum_{n=0}^{N} a_{n} e^{-i \omega_{n} t}\left(\frac{R_{0}}{r}\right)^{n+1} P_{n}(\mu),
$$

where $a_{n}$ is a constant coefficient to be found. The fact that Eq. (8) satisfies Eq. (7) can be checked by direct substitution.

Applying the curl operator to both sides of Eq. (3) and substituting Eq. (4) for $\boldsymbol{v}_{1}$, one obtains

$$
\left(\Delta-\frac{1}{v} \frac{\partial}{\partial t}\right) \psi_{1}=0 .
$$

In view of Eqs. (1) and (6), a solution to Eq. (9) is given by

$$
\boldsymbol{\psi}_{1}=\boldsymbol{e}_{\varepsilon} \sum_{n=1}^{N} b_{n} e^{-i \omega_{n} t} h_{n}^{(1)}\left(k_{n} r\right) P_{n}^{1}(\mu),
$$


where $h_{n}^{(1)}$ is the spherical Hankel function of the first kind, $P_{n}^{1}$ is the associated Legendre polynomial of the first order, $k_{n}=$ $(1+i) / \delta_{n}$, where $\delta_{n}=\sqrt{2 v / \omega_{n}}$ is the viscous penetration depth at frequency $\omega_{n}$, and $b_{n}$ is a constant coefficient to be found.

The components of $\boldsymbol{v}_{1}$ are calculated by

$$
\begin{aligned}
v_{1 r}= & -\frac{1}{R_{0}} \sum_{n=0}^{N}(n+1) e^{-i \omega_{n} t}\left[a_{n}\left(\frac{\bar{x}_{n}}{x_{n}}\right)^{n+2}\right. \\
& \left.+n b_{n} \frac{\bar{x}_{n}}{x_{n}} h_{n}^{(1)}\left(x_{n}\right)\right] P_{n}(\mu), \\
v_{1 \theta}= & \frac{1}{R_{0}} \sum_{n=1}^{N} e^{-i \omega_{n} t}\left\{a_{n}\left(\frac{\bar{x}_{n}}{x_{n}}\right)^{n+2}\right. \\
& \left.-b_{n} \frac{\bar{x}_{n}}{x_{n}}\left[h_{n}^{(1)}\left(x_{n}\right)+x_{n} h_{n}^{(1) /}\left(x_{n}\right)\right]\right\} P_{n}^{1}(\mu),
\end{aligned}
$$

where $x_{n}=k_{n} r, \bar{x}_{n}=k_{n} R_{0}$, and $h_{n}^{(1) /}\left(x_{n}\right)=d h_{n}^{(1)}\left(x_{n}\right) / d x_{n}$. Note that when calculating Eqs. (11) and (12), we have used mathematical properties of $P_{n}(\mu)$ and $P_{n}^{1}(\mu)[19,20]$.

To find $a_{n}$ and $b_{n}$, boundary conditions at the bubble surface are applied. The boundary condition for the first-order liquid velocity requires that the normal component of $\boldsymbol{v}_{1}$ at $r=R_{0}$ be equal to the normal component of the velocity of the bubble surface. This condition is written as

$$
\left.v_{1 r}\right|_{r=R_{0}}=\frac{d r_{s}}{d t}=-i \sum_{n=0}^{N} \omega_{n} s_{n} e^{-i \omega_{n} t} P_{n}(\mu) .
$$

Note that the velocity of the driving acoustic wave is neglected in Eq. (13) because it is assumed small compared to the velocity of the scattered wave, and the translational velocity of the bubble is considered with respect to the velocity of the driving wave.

Substitution of Eq. (11) into Eq. (13) yields

$$
\begin{aligned}
a_{0} & =i R_{0} \omega_{0} s_{0} \\
a_{n}+n h_{n}^{(1)}\left(\bar{x}_{n}\right) b_{n} & =\frac{i R_{0} \omega_{n} s_{n}}{n+1} \quad \text { for } n \geqslant 1 .
\end{aligned}
$$

The choice of the second boundary condition depends on the behavior of the gas-liquid interface. If we deal with a gas bubble without a shell or contamination on the gas-liquid interface, we can apply the condition of slippage [21]. This condition assumes that on the bubble surface the tangential stress vanishes,

$$
\sigma_{r \theta}=\eta\left(\frac{1}{r} \frac{\partial v_{1 r}}{\partial \theta}+\frac{\partial v_{1 \theta}}{\partial r}-\frac{v_{1 \theta}}{r}\right)=0 \text { at } r=R_{0},
$$

where $\sigma_{r \theta}$ denotes the first-order tangential stress [18]. Since in our experiments we deal with pure gas bubbles, we apply Eq. (16).

Substitution of Eqs. (11) and (12) into Eq. (16) provides

$$
\begin{aligned}
& 2(n+2) a_{n}+\left[(n-1)(n+2) h_{n}^{(1)}\left(\bar{x}_{n}\right)-\bar{x}_{n}^{2} h_{n}^{(1) / /}\left(\bar{x}_{n}\right)\right] b_{n} \\
& \quad=0 \quad \text { for } n \geqslant 1 .
\end{aligned}
$$

From Eqs. (15) and (17) it follows that

$$
a_{n}=\frac{i R_{0} \omega_{n} s_{n}\left[\bar{x}_{n}^{2} h_{n}^{(1) / /}\left(\bar{x}_{n}\right)-(n-1)(n+2) h_{n}^{(1)}\left(\bar{x}_{n}\right)\right]}{(n+1)\left[\bar{x}_{n}^{2} h_{n}^{(1) / /}\left(\bar{x}_{n}\right)+\left(n^{2}+3 n+2\right) h_{n}^{(1)}\left(\bar{x}_{n}\right)\right]}
$$

for $n \geqslant 1$,

$$
b_{n}=\frac{2 i R_{0}(n+2) \omega_{n} s_{n}}{(n+1)\left[\bar{x}_{n}^{2} h_{n}^{(1) / /}\left(\bar{x}_{n}\right)+\left(n^{2}+3 n+2\right) h_{n}^{(1)}\left(\bar{x}_{n}\right)\right]}
$$

for $n \geqslant 1$.

Equations (14), (18), and (19) express the linear velocity potentials in terms of the complex amplitudes of the bubble oscillation modes. They will be used below as input data in the equations of acoustic streaming.

\section{B. Equations of acoustic streaming}

Taking the nonlinear incompressible Navier-Stokes equations [18] up to second-order terms with respect to the linear solutions and averaging over time, one obtains

$$
\begin{gathered}
\nabla \cdot\left\langle\boldsymbol{v}_{2}\right\rangle=0 \\
\eta \Delta\left\langle\boldsymbol{v}_{2}\right\rangle-\nabla\left\langle p_{2}\right\rangle=\rho\left\langle\boldsymbol{v}_{1} \cdot \nabla \boldsymbol{v}_{1}\right\rangle,
\end{gathered}
$$

where $\left\langle\boldsymbol{v}_{2}\right\rangle$ and $\left\langle p_{2}\right\rangle$ are the time-averaged second-order velocity and pressure, respectively, and \langle\rangle means the time average.

To satisfy Eq. (20) and the condition of axial symmetry, $\left\langle\boldsymbol{v}_{2}\right\rangle$ is defined by

$$
\left\langle\boldsymbol{v}_{2}\right\rangle=\nabla \times\left\langle\boldsymbol{\psi}_{2}\right\rangle
$$

with

$$
\left\langle\boldsymbol{\psi}_{2}\right\rangle=\left\langle\psi_{2}(r, \theta)\right\rangle \boldsymbol{e}_{\varepsilon} .
$$

Calculating the curl of both sides of Eq. (21) and substituting Eq. (22), one obtains

$$
\Delta^{2}\left\langle\boldsymbol{\psi}_{2}\right\rangle=-\frac{1}{v} \nabla \times\left\langle\boldsymbol{v}_{1} \cdot \nabla \boldsymbol{v}_{1}\right\rangle .
$$

Equation (24) recovers the vorticity equation derived by Westervelt for the case of solenoidal first-order motion [22]; see Eq. (17) in his work.

The vector equation (24) can be transformed (see Appendix A) to an equation for the scalar quantity $\left\langle\psi_{2}\right\rangle$, which was introduced by Eq. (23):

$$
\begin{aligned}
\left(\Delta_{r} \theta\right. & \left.-\frac{1}{r^{2} \sin ^{2} \theta}\right)^{2}\left\langle\psi_{2}\right\rangle \\
= & \frac{1}{2 \nu r} \operatorname{Re}\left\{\frac{\partial}{\partial r}\left[r v_{1 r}\left(\Delta_{r \theta} \psi_{1}^{*}-\frac{\psi_{1}^{*}}{r^{2} \sin ^{2} \theta}\right)\right]\right. \\
& \left.+\frac{\partial}{\partial \theta}\left[v_{1 \theta}\left(\Delta_{r \theta} \psi_{1}^{*}-\frac{\psi_{1}^{*}}{r^{2} \sin ^{2} \theta}\right)\right]\right\} .
\end{aligned}
$$


Here, Re means "the real part of," the asterisk denotes the complex conjugate, and the operator $\Delta_{r \theta}$ is defined by Eq. (A9) in Appendix A.

\section{Equations of microstreaming produced by modes $\boldsymbol{n}$ and $\boldsymbol{m}$}

Time averaging leads to the result that nonzero contributions to acoustic streaming can come either from pairs of modes that oscillate at the same frequency or from the interaction of a mode with itself. Let us assume that modes $n$ and $m$ oscillate at the same frequency and apply Eq. (25) to these two modes. This means that we assume

$$
\boldsymbol{\psi}_{1}=\boldsymbol{\psi}_{1 n}+\boldsymbol{\psi}_{1 m}, \quad \boldsymbol{v}_{1}=\boldsymbol{v}_{1 n}+\boldsymbol{v}_{1 m}, \quad \omega_{n}=\omega_{m} .
$$

In this case, Eq. (9) gives $\Delta \boldsymbol{\psi}_{1}=-k_{n}^{2} \boldsymbol{\psi}_{1}$, which, upon substituting into Eq. (A11), leads to

$$
\Delta_{r \theta} \psi_{1}-\frac{\psi_{1}}{r^{2} \sin ^{2} \theta}=-k_{n}^{2} \psi_{1} .
$$

On substitution of Eq. (27), Eq. (25) reduces to

$$
\begin{aligned}
& \left(\Delta_{r \theta}-\frac{1}{r^{2} \sin ^{2} \theta}\right)^{2}\left\langle\psi_{2}\right\rangle \\
& =\frac{1}{2 v r} \operatorname{Re}\left\{k_{n}^{2}\left[\frac{\partial\left(r v_{1 r} \psi_{1}^{*}\right)}{\partial r}+\frac{\partial\left(v_{1 \theta} \psi_{1}^{*}\right)}{\partial \theta}\right]\right\},
\end{aligned}
$$

where we have used the fact that $k_{n}^{2 *}=-k_{n}^{2}$.

Equations (26) suggest that $\left\langle\psi_{2}\right\rangle$ in Eq. (28) can be divided into three parts:

$$
\left\langle\psi_{2}\right\rangle=\left\langle\psi_{2}^{n m}\right\rangle+\left\langle\psi_{2}^{n n}\right\rangle+\left\langle\psi_{2}^{m m}\right\rangle
$$

where $\left\langle\psi_{2}^{n m}\right\rangle$ is produced by the interaction of modes $n$ and $m$, $\left\langle\psi_{2}^{n n}\right\rangle$ is produced by mode $n$ alone, and $\left\langle\psi_{2}^{m m}\right\rangle$ is produced by mode $m$ alone. It follows from Eq. (28) that the terms of Eq. (29) should obey the following equations:

$$
\begin{gathered}
\left(\Delta_{r \theta}-\frac{1}{r^{2} \sin ^{2} \theta}\right)^{2}\left\langle\psi_{2}^{n m}\right\rangle=\frac{1}{2 v r} \operatorname{Re}\left\{k_{n}^{2}\left[\frac{\partial}{\partial r}\left(r v_{1 n r} \psi_{1 m}^{*}+r v_{1 m r} \psi_{1 n}^{*}\right)+\frac{\partial}{\partial \theta}\left(v_{1 n \theta} \psi_{1 m}^{*}+v_{1 m \theta} \psi_{1 n}^{*}\right)\right]\right\}, \\
\left(\Delta_{r \theta}-\frac{1}{r^{2} \sin ^{2} \theta}\right)^{2}\left\langle\psi_{2}^{n n}\right\rangle=\frac{1}{2 \nu r} \operatorname{Re}\left\{k_{n}^{2}\left[\frac{\partial}{\partial r}\left(r v_{1 n r} \psi_{1 n}^{*}\right)+\frac{\partial}{\partial \theta}\left(v_{1 n \theta} \psi_{1 n}^{*}\right)\right]\right\} .
\end{gathered}
$$

The equation for $\left\langle\psi_{2}^{m m}\right\rangle$ is obtained replacing $n$ by $m$ in Eq. (31).

Substituting the explicit expressions for $\psi_{1 n}, \psi_{1 m}$, and the velocity components into Eqs. (30) and (31), we obtain final equations for the terms of Eq. (29):

$$
\begin{aligned}
\left(\Delta_{r \theta}-\frac{1}{r^{2} \sin ^{2} \theta}\right)^{2}\left\langle\psi_{2}^{n m}\right\rangle= & \frac{n+1}{2 v r^{2}} P_{n}(\mu) P_{m}^{1}(\mu) \operatorname{Re}\left\{k_{n}{ }^{2} a_{n} b_{m}^{*}\left(\frac{\bar{x}_{n}}{x_{n}}\right)^{n+1}\left[(n+1) h_{m}^{(1)}\left(x_{n}\right)-x_{n} h_{m}^{(1) /}\left(x_{n}\right)\right]^{*}\right. \\
& \left.-n k_{n}{ }^{2} b_{n} b_{m}^{*}\left[x_{n} h_{n}^{(1) /}\left(x_{n}\right) h_{m}^{(1) *}\left(x_{n}\right)+x_{n}^{*} h_{n}^{(1)}\left(x_{n}\right) h_{m}^{(1) / *}\left(x_{n}\right)\right]\right\} \\
& +\frac{m+1}{2 v r^{2}} P_{m}(\mu) P_{n}^{1}(\mu) \operatorname{Re}\left\{k_{n}{ }^{2} a_{m} b_{n}^{*}\left(\frac{\bar{x}_{n}}{x_{n}}\right)^{m+1}\left[(m+1) h_{n}^{(1)}\left(x_{n}\right)-x_{n} h_{n}^{(1) /}\left(x_{n}\right)\right]^{*}\right. \\
& \left.-m k_{n}{ }^{2} b_{m} b_{n}^{*}\left[x_{n} h_{m}^{(1) /}\left(x_{n}\right) h_{n}^{(1) *}\left(x_{n}\right)+x_{n}^{*} h_{m}^{(1)}\left(x_{n}\right) h_{n}^{(1) / *}\left(x_{n}\right)\right]\right\} \\
& -\frac{\sqrt{1-\mu^{2}}}{2 v r^{2}}\left[P_{n}^{1}(\mu) P_{m}^{1}(\mu)\right]^{\prime} \operatorname{Re}\left\{b_{m}^{*} k_{n}^{2} h_{m}^{(1) *}\left(x_{n}\right)\left[a_{n}\left(\frac{\bar{x}_{n}}{x_{n}}\right)^{n+1}-b_{n}\left[h_{n}^{(1)}\left(x_{n}\right)+x_{n} h_{n}^{(1) /}\left(x_{n}\right)\right]\right]\right. \\
+ & \left.b_{n}^{*} k_{n}^{2} h_{n}^{(1) *}\left(x_{n}\right)\left[a_{m}\left(\frac{x_{n}}{x_{n}}\right)^{m+1}-b_{m}\left[h_{m}^{(1)}\left(x_{n}\right)+x_{n} h_{m}^{(1) /}\left(x_{n}\right)\right]\right]\right\} \\
\left.\frac{1}{r^{2} \sin ^{2} \theta}\right)^{2}\left\langle\psi_{2}^{n n}\right\rangle & =\frac{n+1}{2 v r^{2}} P_{n}(\mu) P_{n}^{1}(\mu)\left(\frac{x_{n}}{x_{n}}\right)^{n+1} \operatorname{Re}\left\{k_{n}{ }^{2} a_{n} b_{n}^{*}\left[(n+1) h_{n}^{(1)}\left(x_{n}\right)-x_{n} h_{n}^{(1) /}\left(x_{n}\right)\right]^{*}\right\} \\
& -\frac{\sqrt{1-\mu^{2}}}{\nu r^{2}} P_{n}^{1}(\mu) P_{n}^{1 /}(\mu) \operatorname{Re}\left\{k_{n}^{2} h_{n}^{(1) *}\left(x_{n}\right)\left[a_{n} b_{n}^{*}\left(\frac{\bar{x}_{n}}{x_{n}}\right)^{n+1}-b_{n} b_{n}^{*} x_{n} h_{n}^{(1) /}\left(x_{n}\right)\right]\right\} .
\end{aligned}
$$

In the next section, Eq. (32) will be applied to the case $n=0$. In the second part of our study, Eqs. (32) and (33) will be applied to the case $n=1$.

\section{Microstreaming produced by modes 0 and $m$}

The mathematical complexity of Eqs. (32) and (33) makes it difficult to solve them at arbitrary values of $n$ and $m$. To make the problem amenable to analytical solution, we consider an important practical case where acoustic microstreaming is generated as a result of the interaction of mode 0 with a mode of order $m \geqslant 1$. 


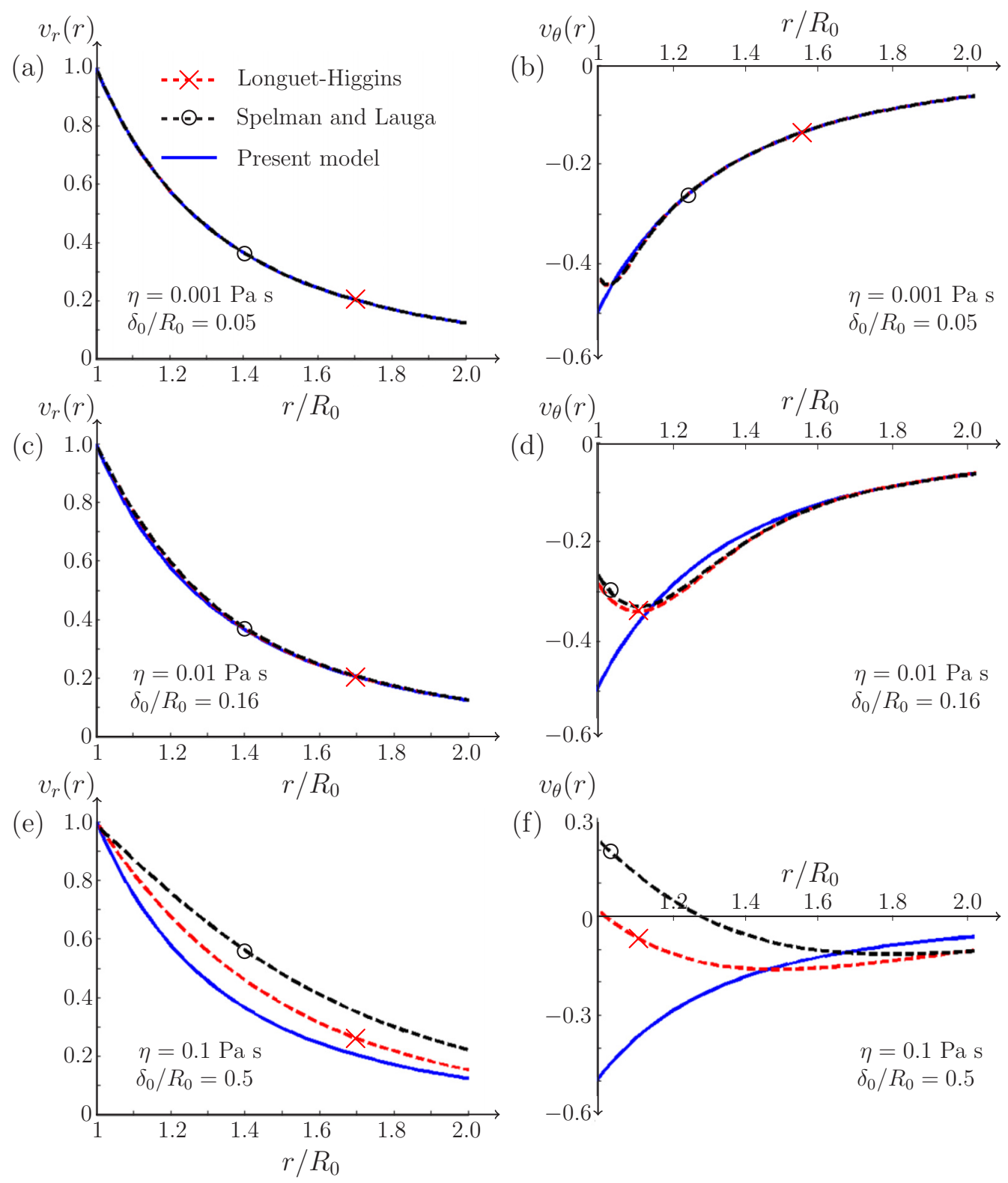

FIG. 2. Comparison of the present model to the models of Longuet-Higgins [7] and Spelman and Lauga [10] for the linear liquid velocity produced by the translational motion of the bubble. Left column: $r$ dependence of the radial linear velocity. Right column: $r$ dependence of the tangential linear velocity. The subfigures compare the behavior of the velocity components given by the three models at increasing values of the liquid viscosity: (a, b) $\eta=0.001 \mathrm{~Pa} \mathrm{~s}$; (c, d) $\eta=0.01 \mathrm{~Pa} \mathrm{~s}$; (e, f) $\eta=0.1 \mathrm{~Pa}$ s. Distinctions between the models increase with increasing ratio $\delta_{0} / R_{0}$.

Setting $n=0$ in Eq. (32), we obtain

$$
\begin{aligned}
& \left(\Delta_{r \theta}-\frac{1}{r^{2} \sin ^{2} \theta}\right)^{2}\left\langle\psi_{2}^{0 m}\right\rangle \\
& \quad=-\frac{R_{0}}{2 v r^{3}} P_{m}^{1}(\mu) \operatorname{Re}\left\{k_{0}^{2} a_{0}^{*} b_{m}\left[h_{m}^{(1)}\left(k_{0} r\right)-k_{0} r h_{m}^{(1) /}\left(k_{0} r\right)\right]\right\} .
\end{aligned}
$$

Solving Eq. (34) requires cumbersome calculations. Therefore, they are performed in Appendix B. As a result, the solution of Eq. (34) is found to be

$$
\left\langle\psi_{2}^{0 m}\right\rangle=-\frac{R_{0}}{2 v} P_{m}^{1}(\mu) \operatorname{Re}\left\{k_{0} a_{0}^{*} b_{m} F_{m}\left(k_{0} r\right)\right\}
$$

where the function $F_{m}\left(k_{0} r\right)$ is defined by Eq. (B12). Equation (35) leads to the following expressions for the components of the Eulerian streaming velocity:

$$
\begin{gathered}
\left\langle v_{2 r}^{0 m}\right\rangle=\frac{m(m+1) R_{0}}{2 v r} P_{m}(\mu) \operatorname{Re}\left\{k_{0} a_{0}^{*} b_{m} F_{m}\left(k_{0} r\right)\right\}, \\
\left\langle v_{2 \theta}^{0 m}\right\rangle=\frac{R_{0}}{2 v r} P_{m}^{1}(\mu) \operatorname{Re}\left\{k_{0} a_{0}^{*} b_{m}\left[F_{m}\left(k_{0} r\right)+k_{0} r F_{m}^{\prime}\left(k_{0} r\right)\right]\right\},
\end{gathered}
$$

where the function $F_{m}^{/}\left(k_{0} r\right)$ is defined by Eq. (B21).

In the process of calculating Eqs. (36) and (37), we have also obtained the Stokes drift velocity [7]. Its components are given by Eqs. (B32) and (B33). The summation of the 
Eulerian streaming velocity and the Stokes drift velocity provides the Lagrangian streaming velocity, which makes our derivation complete. A MATLAB code for the calculation of the Eqs. (36), (37), (B32), and (B33) is provided as Supplemental Material [23].

\section{E. Comparison with previous theories}

Streaming produced by modes 0 and 1 was first derived by Longuet-Higgins [7]. This case was later included in the arbitrary-mode analysis performed by Spelman and Lauga [10]. Before comparing our results to the above studies, it is worth noting that in both of them the physical situation is different from ours. Both studies assume that the bubble is fixed while the liquid oscillates about it, whereas we assume that the bubble is moving while the liquid at infinity is at rest. This means that, in their case, at infinity the amplitude of the first-order liquid velocity tends to a nonzero constant, as expressed by a term with $r^{2}$ dependence in the linear stream function $\Psi_{(\mathrm{LH})}^{10}$ derived by Longuet-Higgins,

$$
\begin{aligned}
\Psi_{(\mathrm{LH})}^{10}(r, \mu)= & {\left[\frac{1}{2}\left(r^{2}-\frac{1}{r}\right)+\frac{B}{\alpha^{2}} \frac{1}{r}+\frac{C}{\alpha^{2}}\left(1+\frac{1}{\alpha r}\right) e^{-\alpha(r-1)}\right] } \\
& \times(1-\mu),
\end{aligned}
$$

where $\alpha=(1+i) R_{0} / \delta$, and $B, C$ are coefficients given in Ref. [7]. This $r^{2}$ term then leads to a term with $r^{-3}$ dependence in the Stokes drift velocity (see Eq. (8.16) of Ref. [7]). As far as linear solutions are concerned, the two physically different situations lead to mathematically identical equations. However, for second-order solutions, that is not the case. This is seen, for example, if one compares the Stokes drift given by Longuet-Higgins and Eqs. (B32) and (B33) of our study: the $r^{-3}$ dependence obtained by Longuet-Higgins is impossible if the liquid is at rest at infinity. Therefore, our results for streaming produced by modes 0 and 1 cannot be transformed to those of Longuet-Higgins. The same is true when our work is compared to that of Spelman and Lauga [10], who derived the full microstreaming velocity accounting for arbitrary axisymmetric shape modes using the same mathematical approach as Longuet-Higgins [7]. Analysis of the $0-1$ interaction performed by Spelman and Lauga [10] leads to the following linear stream function:

$$
\begin{aligned}
\Psi_{(\mathrm{SL})}^{10}(r, \mu)= & D_{0}(1-\mu)-\frac{1}{2}\left[B_{1} \frac{\sqrt{r}}{\alpha^{2}} K_{\frac{3}{2}}(\alpha r)+\frac{D_{1}}{r}\right] \\
& \times \sqrt{1-\mu^{2}} P_{1}^{1}(\mu),
\end{aligned}
$$

where $\alpha=(1+i) / \delta, K_{n}(x)$ is the modified Bessel function of the second kind of order $n$, and $B_{0}, B_{1}, D_{1}$ are coefficients given in Ref. [10]. This stream function also leads to a constant linear velocity at infinity through the term $D_{0}(1-\mu)$.

Before we go to comparing streaming velocities, it is instructive to compare the linear velocities given by our approach, which impose no restrictions on the viscous penetration depth, with those given by Longuet-Higgins' and Spelman and Lauga's approaches based on the approximation of a thin viscous boundary layer. This comparison gives an idea of a range within which the approximation of a thin viscous boundary layer is valid. In order to allow the comparison, we have eliminated the $r^{2}$ term in Eq. (38) and the term $D_{0}(1-\mu)$ in Eq. (39). Numerical simulations were performed for the radial and tangential velocity components at various values of the liquid viscosity. In experiments on bubble dynamics, water-glycerin mixtures are used, so the liquid viscosity can vary from $0.001 \mathrm{~Pa} \mathrm{~s}$ (pure water) to $1.48 \mathrm{~Pa}$ s (pure glycerin). We made calculations for viscosities within this range at $R_{0}=50 \mu \mathrm{m}$ and $f=50 \mathrm{kHz}$. The results are presented in Fig. 2 . Note that the velocity components are normalized by the factor $\omega_{0}\left|s_{1}\right|$.

Figure 2 shows that at $\eta=0.001 \mathrm{~Pa}$ s (water), a difference between the predictions of the models is insignificant. The radial velocities are identical, and the tangential ones demonstrate a small distinction. However, when the liquid viscosity (and accordingly, the ratio of the viscous penetration depth $\delta_{0}$ to the bubble radius $R_{0}$ ) is increased, a quite considerable difference between the tangential velocities appears, though the value of the viscosity is still small compared to the viscosity of glycerin. The variation of the liquid viscosity affects the tangential velocity more strongly than the radial one because the latter obeys the no-penetration boundary condition at the bubble surface, which acts in both perfect and viscous liquid, whereas the tangential

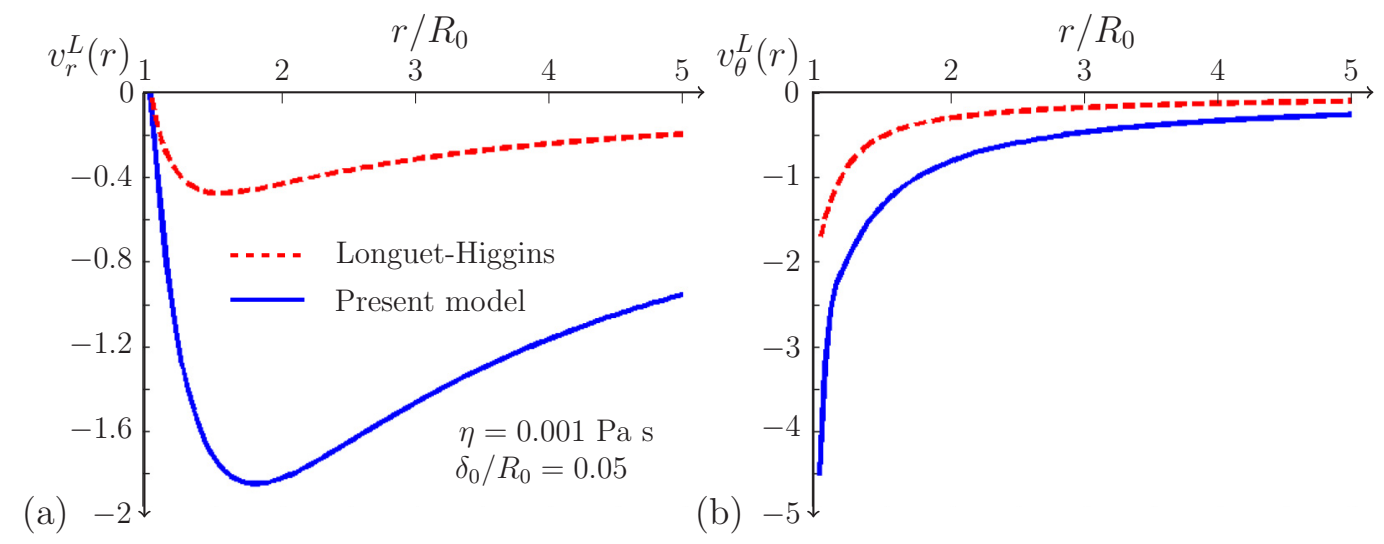

FIG. 3. The $r$ dependence of the radial (a) and tangential (b) components of the Lagrangian streaming velocity given by Longuet-Higgins' theory [7] and the present model for modes 0 and 1. 


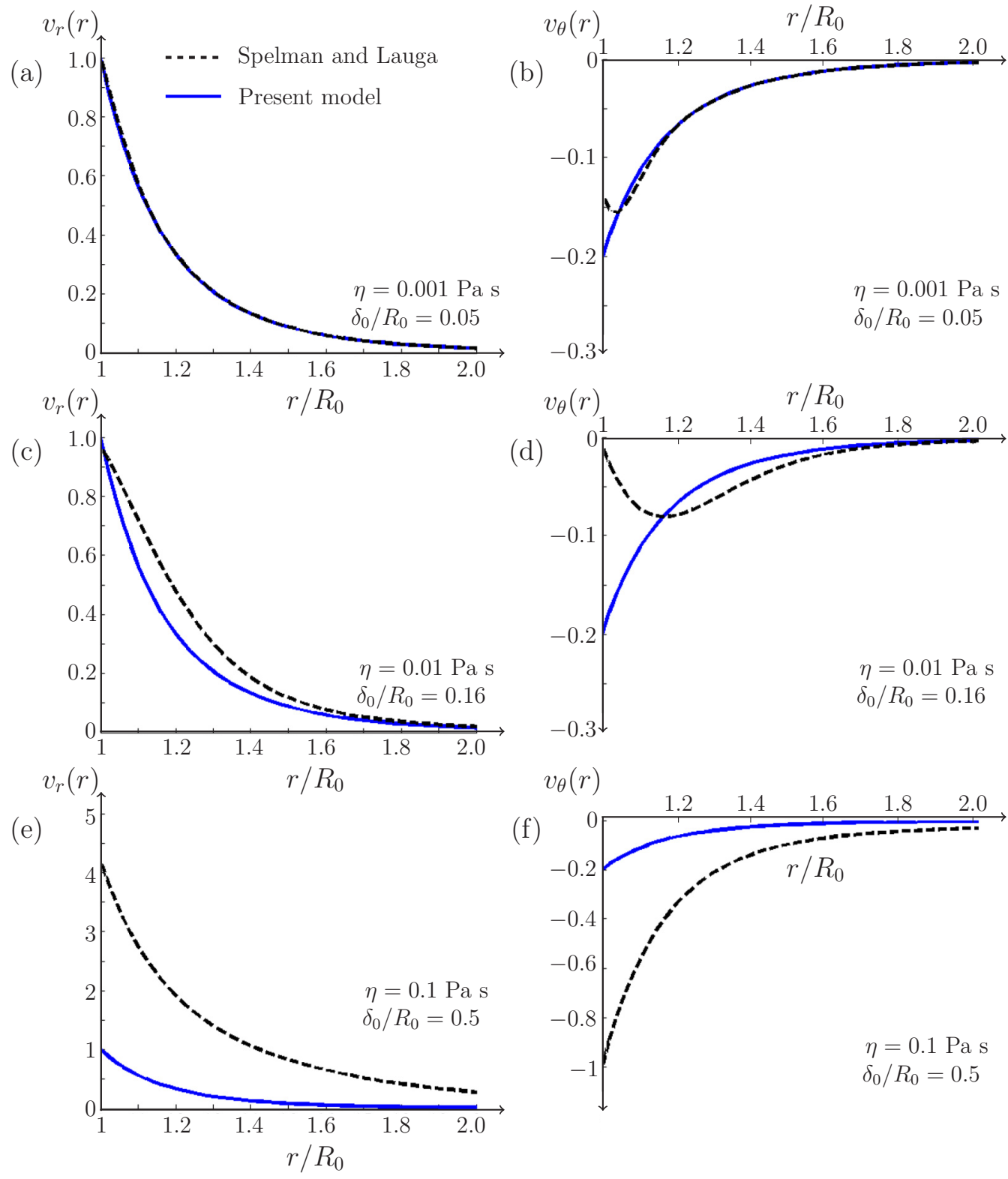

FIG. 4. Comparison of the present model to the theory of Spelman and Lauga [10] for the linear liquid velocity produced by mode 4 at various values of the liquid viscosity. Left column: $r$ dependence of the radial linear velocity. Right column: $r$ dependence of the tangential linear velocity. Upper line: $\eta=0.001 \mathrm{~Pa}$. Middle line: $\eta=0.01 \mathrm{~Pa}$ s. Bottom line: $\eta=0.1 \mathrm{~Pa}$ s.

velocity obeys the boundary condition of zero tangential stress at the bubble surface, which appears only in a viscous liquid. Therefore, the difference between the models, which increases with increasing viscosity, has a stronger effect on the tangential velocity, as Fig. 2(f) demonstrates.

When considering the Lagrangian streaming velocity for the $0-1$ interaction, we compare our results only to those of Longuet-Higgins (Eqs. (9.1) and (9.2) of Ref. [7]), as the model of Spelman and Lauga [10] leads to the same result for the lowest-order Lagrangian streaming in the limit of small viscosity. The calculations were made at the following values of physical parameters: $\rho=1000 \mathrm{~kg} / \mathrm{m}^{3}, \eta=0.001$ Pa s, $R_{0}=50 \mu \mathrm{m}$, and $f=50 \mathrm{kHz}$. The phase shift between the modes was set to $\pi / 2$. The velocities are normalized by the factor $\omega_{0}\left|s_{0}\right|\left|s_{1}\right| / R_{0}$. Figure 3 shows the $r$ dependence of the velocity components. As one can see, our theory predicts higher velocity amplitudes. However, it should be emphasized once again that Fig. 3 in fact compares two different physical cases.

In order to illustrate the interaction between the radial mode and a surface mode with $n>1$ oscillating at the same frequency, our model is compared to that of Spelman and Lauga [10]. First, the evolution of the $r$ dependence of the radial and tangential linear velocities is shown in Fig. 4 for increasing values of the liquid viscosity and the ratio of the viscous penetration depth to the bubble radius. While both models show similar behavior for low viscosity, a considerable difference arises for higher viscosities. Figure 5 compares the components of the Lagrangian streaming velocity given by our model and that of 


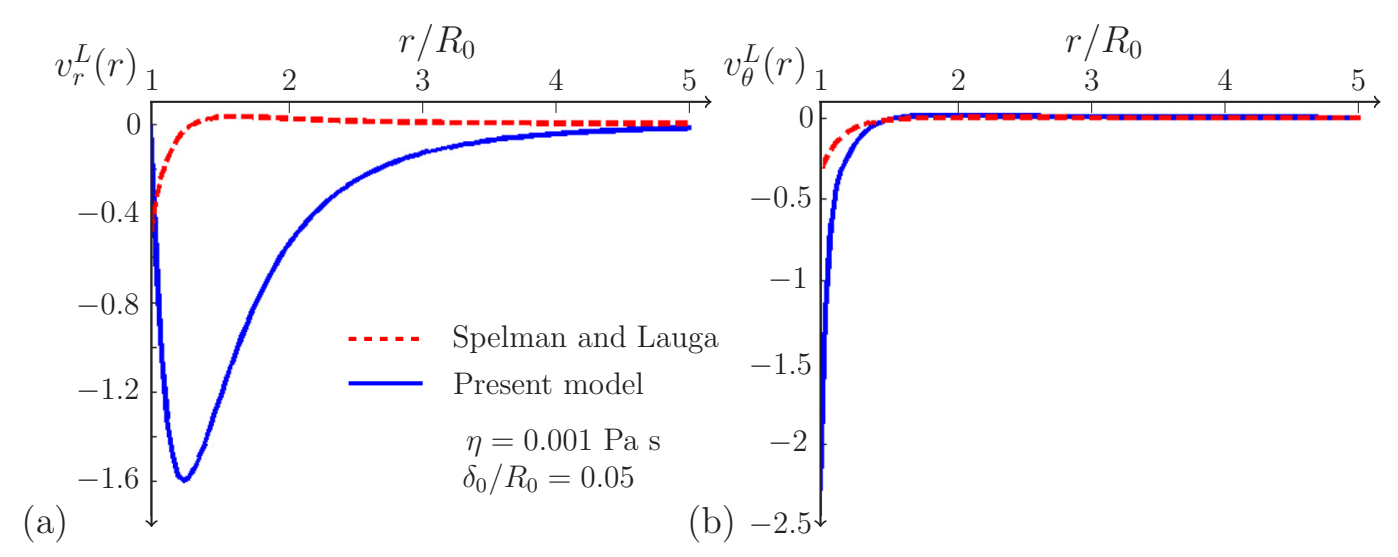

FIG. 5. The $r$ dependence of the radial (a) and tangential (b) components of the Lagrangian streaming velocity given by the theory of Spelman and Lauga [10] and the present model for modes 0 and 4.

Spelman and Lauga [10] at $\eta=0.001 \mathrm{~Pa}$ s. As in the case $0-1$, our model predicts streaming velocities with higher amplitudes.

(a)

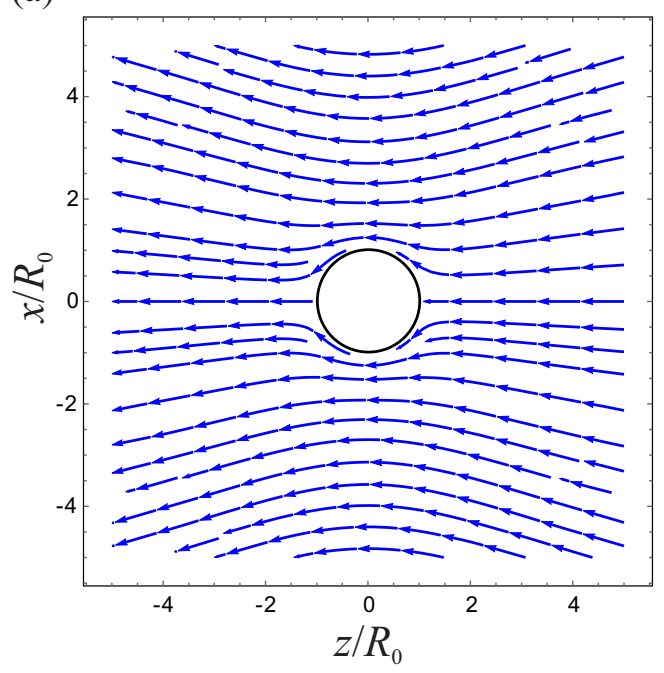

(c)

Modes 0 and 3

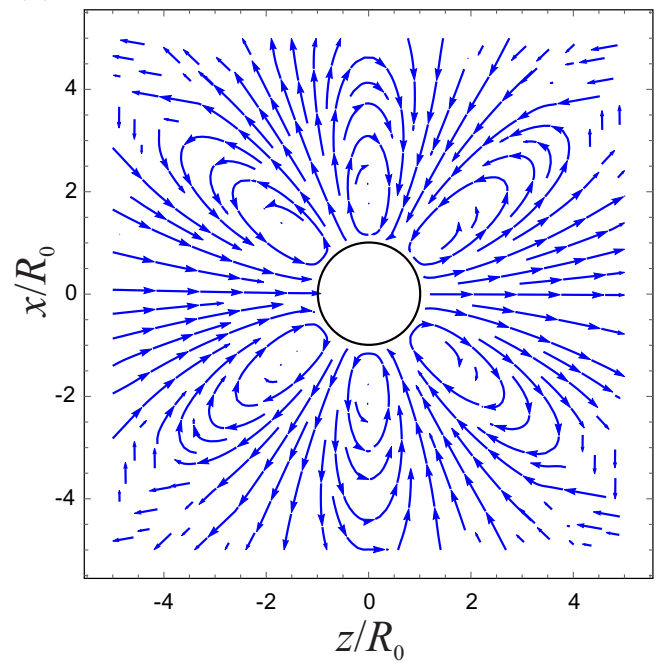

\section{NUMERICAL AND EXPERIMENTAL RESULTS}

Figure 6 presents examples of numerical streamline patterns produced by modes $0-1,0-2,0-3$, and $0-4$. The

(b)

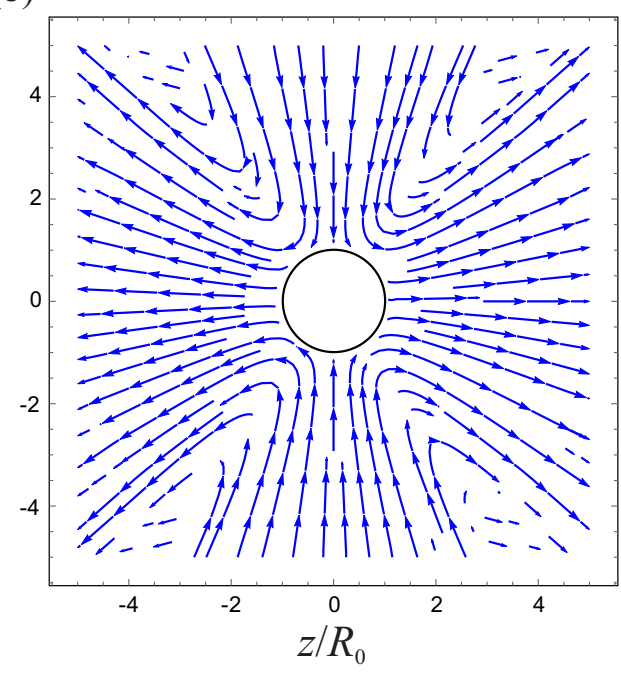

(d)

Modes 0 and 4

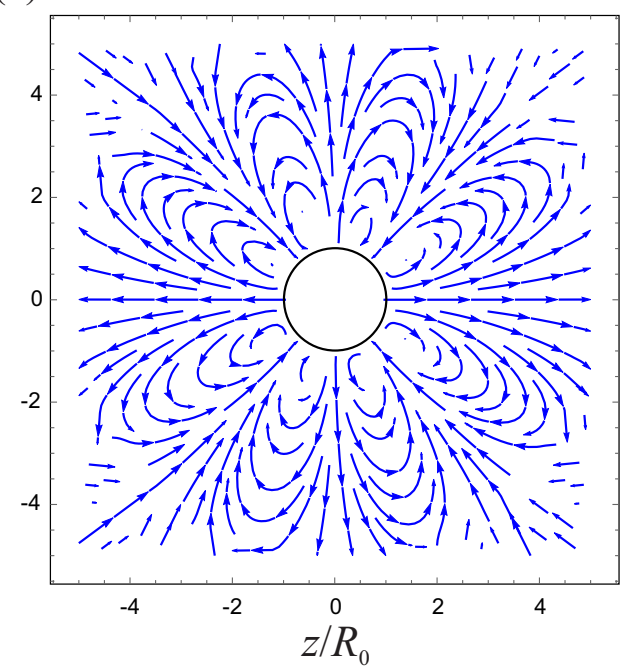

FIG. 6. Numerical examples of streamline patterns produced by various mode pairs: (a) pair 0-1, (b) pair 0-2, (c) pair 0-3, and (d) pair 0-4. 

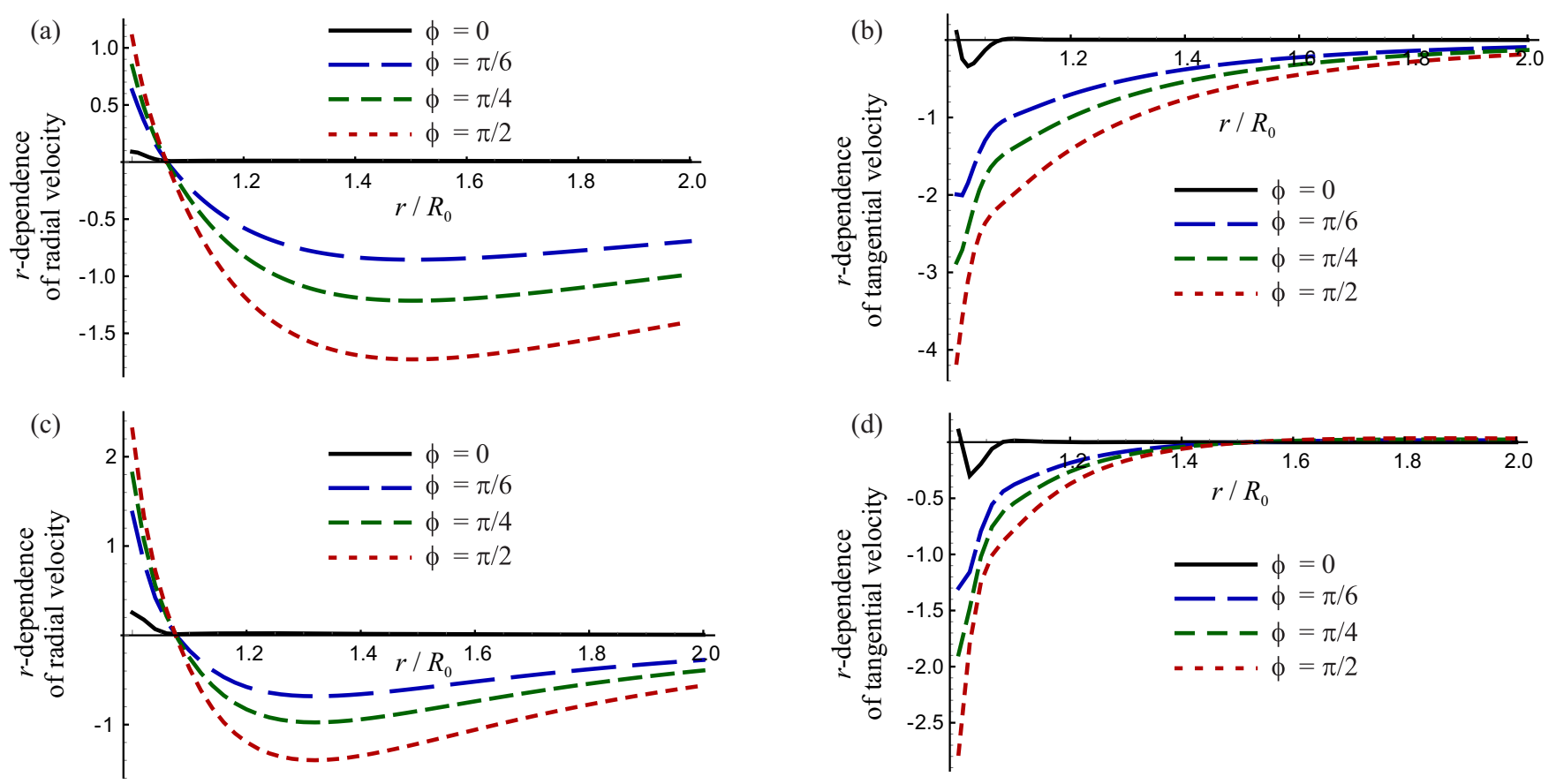

FIG. 7. Dependence of the Eulerian streaming velocity components on the distance from the bubble surface at various values of the phase shift $\phi$ between the modes: (a, b) modes 0 and 2, (c, d) modes 0 and 4.

simulations were made at the following values of physical parameters: $\rho=1000 \mathrm{~kg} / \mathrm{m}^{3}, \eta=0.001 \mathrm{~Pa} \mathrm{~s}, f=50 \mathrm{kHz}$, and $R_{0}=100 \mu \mathrm{m}$. In order to eliminate from consideration the magnitudes of the modes $\left|s_{0}\right|$ and $\left|s_{m}\right|$, the streaming velocity was normalized and made dimensionless by multiplying it by the factor $R_{0} /\left(\omega_{0}\left|s_{0}\right|\left|s_{m}\right|\right)$. The phase shift between modes 0 and 1 is set to $\pi / 2$. For the other mode pairs, the phase shift is zero. Figure 6 shows the streamlines of the Lagrangian velocity. As one can see, main vortices look like lobes whose number equals $2 m$.

Equations (36) and (37) show that the $r$ dependence and the $\theta$ dependence of the streaming velocity components can be separated. The $\theta$ dependence is determined by the angular functions $P_{m}(\mu)$ and $P_{m}^{1}(\mu)$. The remaining terms in Eqs. (36) and (37) determine the $r$ dependence. According to this fact, Fig. 7 shows the $r$ dependence of the Eulerian streaming velocity components at various values of the phase shift between involved modes. It will be recalled that the velocity components are scaled by the factor indicated above. Figures 7(a) and 7(b) illustrate the case of modes 0 and 2. Figures 7(c) and 7 (d) illustrate the case of modes 0 and 4 . In both cases, the amplitude of the streaming velocity increases considerably as the phase shift $\phi$ varies from zero to $\pi / 2$. Another observation is that the velocity amplitude decays within a short distance from the bubble surface, especially at $\phi=0$.

In order to illustrate experimentally these theoretical findings, one needs to observe the microstreaming around a free (far from boundaries) nonspherically oscillating gas bubble and to capture both its interface dynamics and the fluid velocity. Such experimental challenge has been recently overcome and is explained in greater detail elsewhere [24,25]. A short summary of the relevant points is given in the following. The experiments rely on the trapping of micrometric bubbles in a $31.25-\mathrm{kHz}$ standing wave field established within an $8-\mathrm{cm}$ cubic water tank (see Fig. 8). Gas bubbles are induced by short laser pulses and then moved towards pressure antinodes according to acoustic radiation (primary Bjerknes) force. To activate a surface mode, it is necessary to reach bubble size and exceed an acoustic pressure threshold at which the surface instability may develop. The radius of each laser-nucleated bubble lies in the range $20-40 \mu \mathrm{m}$. If desired, a trapped bubble can be grown by multiple coalescences. The coalescence is used as a trigger for nonspherical oscillation when the bubble is driven at sufficiently high acoustic pressure amplitude.

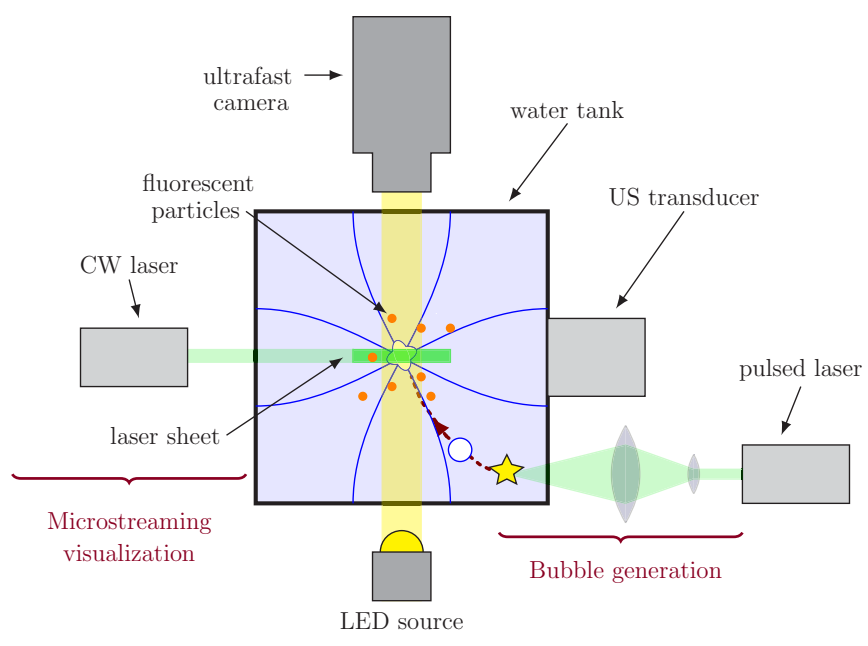

FIG. 8. Schematic representation of the experimental setup including (right part) the system for bubble generation and the ultrasound transducer and (left part) devices for microstreaming visualization. Both bubble dynamics and particle motions are recorded with a high-speed camera illuminated by a LED source (for bubble dynamics) or a laser sheet (for capturing fluorescent particle motion). 
(a)

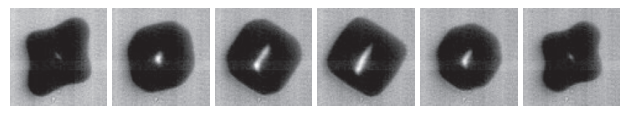

(b)
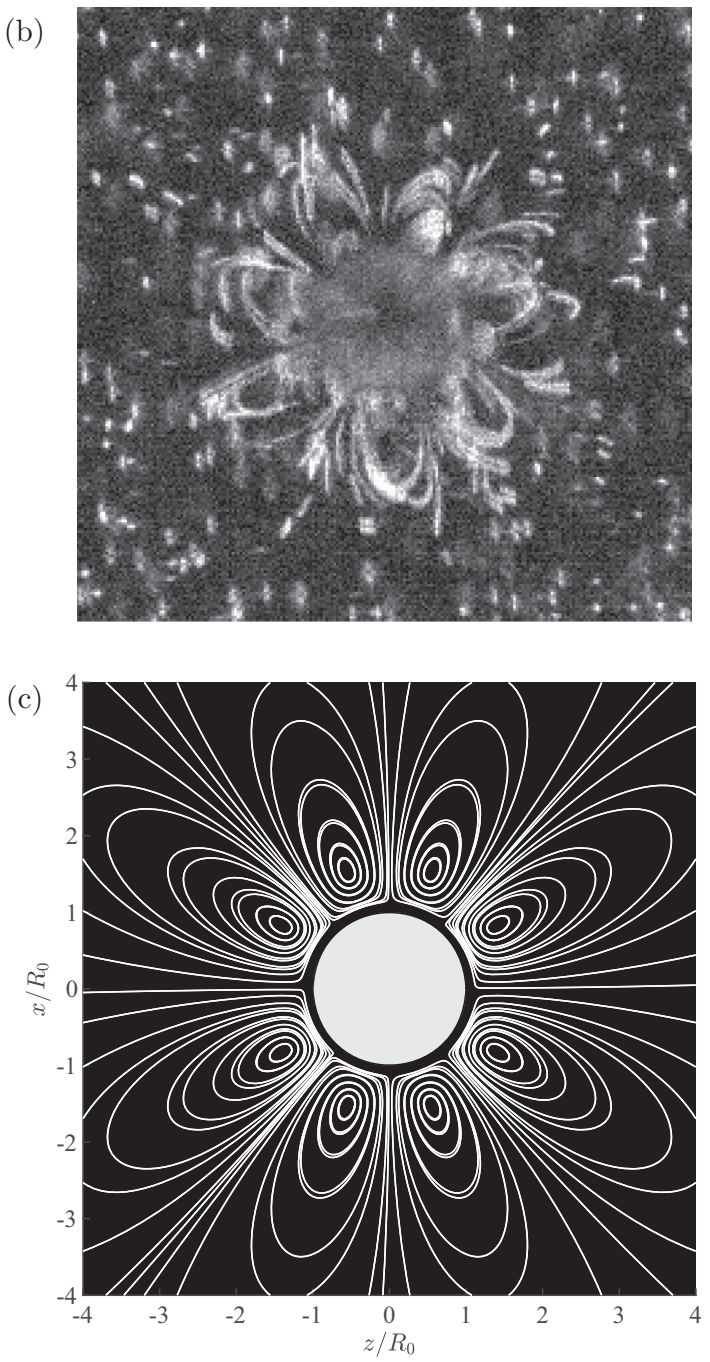

FIG. 9. (a) Snapshot series of the bubble dynamics on one acoustic period corresponding to a bubble of equilibrium radius $R_{0}=53.9 \mu \mathrm{m}$ driven by a $31.25-\mathrm{kHz}$ ultrasound field at acoustic amplitude $P_{a}=25.9 \mathrm{kPa}$ and excited at mode 4 . The time interval between two successive snapshots is $5.55 \mu$ s (image size $160 \times 160$ $\mu \mathrm{m}$ ). (b) Streak photography of the resulting microstreaming pattern (image size $460 \times 460 \mu \mathrm{m}$ ). (c) Theoretical streamline pattern.

Indeed, the coalescence process leads to a highly initial deformed bubble shape, appearing as an initial condition for surface instabilities. According to the value of the equilibrium coalesced bubble radius, the present experimental setup allows triggering modes 2,3 , or 4 . Of interest is that those nonspherical oscillations are obtained in a steady-state regime [26], allowing the development of time-stable microstreaming. For each investigated bubble, both the interface dynamics and the induced microstreaming are alternatively captured by a high-speed camera. According to the coalescence technique, the symmetry axis of the nonspherical oscillations is controlled by the direction of the bubble impact prior to the coalescence. This allows characterizing accurately the bubble interface dynamics in the plane of the camera. The bubble interface can then be decomposed onto a set of Legendre polynomials according to the theoretical assumption expressed by Eq. (1). This decomposition results in the description of the temporal evolution of the radial, $R(t)$, and surface, $a_{n}(t)$, mode amplitudes. To verify the developed theory, we use these experimentally obtained modal amplitudes as input parameters in the developed analytical model. Consequently, the obtained streaming pattern can be compared to the experimentally observed one. As nonspherical oscillations exhibit a parametric behavior, surface mode oscillations are usually oscillating at half the driving frequency when a given mode number is excited on its first parametric resonance. In order to achieve a $0-m$ interaction at the same frequency, one needs to excite a surface mode on its second parametric resonance. Such experimental cases, for which the predominant mode oscillates at the same frequency as the radial mode, are those of mode 4 in our configuration.

Figure 9 presents the experimental and numerical results corresponding to a bubble of radius $R_{0}=53.9 \mu \mathrm{m}$, driven at the acoustic amplitude $P_{a}=25.9 \mathrm{kPa}$. The microstreaming pattern, Fig. 9(b), consists of eight lobes with almost an identical radial extension for each of them. The modal decomposition reveals a predominant fourth mode whose amplitude is measured to be $\left|s_{4}\right|=13 \mu \mathrm{m}$, in addition to the radial mode whose amplitude is about $\left|s_{0}\right|=6 \mu \mathrm{m}$. The phase shift between mode 0 and 4 is found to be $\phi_{04}=-0.95 \pi$. All other modal contents are negligible (it was verified that $\left|s_{n}\right|<$ $3 \mu \mathrm{m}$ for $n \neq 0,4)$; hence the microstreaming is theoretically generated by the interaction of mode 0 and 4 with the two contributions $0-4$ and $4-4$. We recall that the $n-n$ interaction, governed by Eq. (33), is out of the scope of this paper. We therefore compare the experimental results of the streamline pattern to the theoretical one when considering only the $0-4$ interaction. When applying the experimental parameters $\left|s_{0}\right|$, $\left|s_{4}\right|$, and $\phi_{04}$ for the theoretical modeling, the corresponding numerical streamline pattern is presented in Fig. 9(c). This pattern also presents eight lobes with an identical radial extension for each of them and shows satisfying agreement with the experimental case.

\section{CONCLUSIONS}

In the present paper, a theory has been developed that allows one to model the velocity field of acoustic microstreaming produced by nonspherical oscillations of an acoustically driven gas bubble. The theory assumes that some of the bubble oscillation modes are excited parametrically and oscillate at frequencies that can be different from the driving frequency. Analytical solutions are derived in terms of complex amplitudes of oscillation modes, which means that the mode amplitudes are assumed to be known and serve as input data when the velocity field of acoustic microstreaming is calculated. No restrictions are imposed on the ratio of the bubble radius to the viscous penetration depth. The present paper describes the first part of the study in which a general theory is developed and then applied to the case that acoustic microstreaming is generated by the interaction of the breathing mode (mode 0 ) with a mode of arbitrary order $m \geqslant 1$. Numerical simulations 
of streamline patterns show good qualitative agreement with experimental observations.

The developed theory does not consider azimuthal modes, which may appear at shape oscillations of high order due to fluctuations. The occurrence of azimuthal modes breaks the axial symmetry of the problem and makes its analytical consideration much more complicated. A development of the theory in this direction is very challenging and could be the subject of further studies.

\section{ACKNOWLEDGMENT}

This work was supported by the LabEx CeLyA of the University of Lyon (Grant No. ANR-10-LABX-0060/ANR11-IDEX-0007). A.A.D. gratefully acknowledges financial support from Institut National des Sciences Appliquées de Lyon (INSA de Lyon).

\section{APPENDIX A: DERIVATION OF EQ. (25)}

We wish to show how Eq. (25) is derived from Eq. (24).

By substituting Eq. (4) into the right-hand side of Eq. (24), we obtain

$$
\begin{aligned}
\nabla \times\left\langle\boldsymbol{v}_{1} \cdot \nabla \boldsymbol{v}_{1}\right\rangle= & \nabla \times\left\langle\left(\nabla \varphi_{1}\right) \cdot \nabla\left(\nabla \times \boldsymbol{\psi}_{1}\right)\right. \\
& +\left(\nabla \times \boldsymbol{\psi}_{1}\right) \cdot \nabla \nabla \varphi_{1} \\
& \left.+\left(\boldsymbol{\nabla} \times \boldsymbol{\psi}_{1}\right) \cdot \nabla\left(\nabla \times \boldsymbol{\psi}_{1}\right)\right\rangle .
\end{aligned}
$$

We then use the following vector identity [19]:

$$
\boldsymbol{a} \cdot \nabla \boldsymbol{b}+\boldsymbol{b} \cdot \nabla \boldsymbol{a}=\nabla(\boldsymbol{a} \cdot \boldsymbol{b})-\boldsymbol{a} \times(\nabla \times \boldsymbol{b})-\boldsymbol{b} \times(\nabla \times \boldsymbol{a}),
$$

in which we set $\boldsymbol{a}=\nabla \varphi_{1}, \boldsymbol{b}=\nabla \times \boldsymbol{\psi}_{1}$ and substitute the result into Eq. (A1). We obtain

$$
\begin{aligned}
\nabla \times\left\langle\boldsymbol{v}_{1} \cdot \nabla \boldsymbol{v}_{1}\right\rangle= & \nabla \times\left\langle-\left(\nabla \varphi_{1}\right) \times\left[\nabla \times\left(\nabla \times \boldsymbol{\psi}_{1}\right)\right]\right. \\
& \left.+\left(\nabla \times \boldsymbol{\psi}_{1}\right) \cdot \nabla\left(\nabla \times \boldsymbol{\psi}_{1}\right)\right\rangle .
\end{aligned}
$$

It follows from Eq. (6) that $\nabla \cdot \psi_{1}=0$. Therefore, the following identity is valid [19]:

$$
\nabla \times\left(\nabla \times \psi_{1}\right)=\nabla \nabla \cdot \psi_{1}-\Delta \psi_{1}=-\Delta \psi_{1} .
$$

Substitution of Eq. (A4) into Eq. (A3) yields

$$
\begin{aligned}
\nabla \times\left\langle\boldsymbol{v}_{1} \cdot \nabla \boldsymbol{v}_{1}\right\rangle= & \nabla \times\left\langle\left(\nabla \varphi_{1}\right) \times \Delta \boldsymbol{\psi}_{1}\right. \\
& \left.+\left(\boldsymbol{\nabla} \times \boldsymbol{\psi}_{1}\right) \cdot \nabla\left(\nabla \times \boldsymbol{\psi}_{1}\right)\right\rangle .
\end{aligned}
$$

With the help of Eq. (A2), in which $\boldsymbol{a}=\boldsymbol{b}=\boldsymbol{\nabla} \times \boldsymbol{\psi}_{1}$, and Eq. (A4), Eq. (A5) is transformed to

$$
\begin{aligned}
\nabla \times\left\langle\boldsymbol{v}_{1} \cdot \nabla \boldsymbol{v}_{1}\right\rangle= & \nabla \times\left\langle\left(\nabla \varphi_{1}\right) \times \Delta \boldsymbol{\psi}_{1}-\left(\nabla \times \boldsymbol{\psi}_{1}\right)\right. \\
& \left.\times\left[\nabla \times\left(\nabla \times \boldsymbol{\psi}_{1}\right)\right]\right\rangle \\
= & \nabla \times\left\langle\left(\nabla \varphi_{1}\right) \times \Delta \boldsymbol{\psi}_{1}+\left(\nabla \times \boldsymbol{\psi}_{1}\right) \times \Delta \boldsymbol{\psi}_{1}\right\rangle \\
= & \nabla \times\left\langle\boldsymbol{v}_{1} \times \Delta \boldsymbol{\psi}_{1}\right\rangle .
\end{aligned}
$$

Substitution of Eq. (A6) into Eq. (24) results in

$\Delta^{2}\left\langle\boldsymbol{\psi}_{2}\right\rangle=-\frac{1}{v} \nabla \times\left\langle\boldsymbol{v}_{1} \times \Delta \boldsymbol{\psi}_{1}\right\rangle=\frac{1}{2 v} \operatorname{Re}\left\{\nabla \times\left[\left(\Delta \boldsymbol{\psi}_{1}^{*}\right) \times \boldsymbol{v}_{1}\right]\right\}$,

where Re means "the real part of" and the asterisk denotes the complex conjugate.
The Laplace operator $\Delta$ [19] can be represented as follows:

$$
\Delta=\Delta_{r \theta}+\frac{1}{r^{2} \sin ^{2} \theta} \frac{\partial^{2}}{\partial \varepsilon^{2}},
$$

where

$$
\Delta_{r \theta}=\frac{1}{r^{2}} \frac{\partial}{\partial r}\left(r^{2} \frac{\partial}{\partial r}\right)+\frac{1}{r^{2} \sin \theta} \frac{\partial}{\partial \theta}\left(\sin \theta \frac{\partial}{\partial \theta}\right) .
$$

The use of Eq. (23) and the fact that $\partial^{2} \boldsymbol{e}_{\varepsilon} / \partial \varepsilon^{2}=-\boldsymbol{e}_{\varepsilon}$ gives

$$
\Delta^{2}\left\langle\boldsymbol{\psi}_{2}\right\rangle=\boldsymbol{e}_{\varepsilon}\left(\Delta_{r \theta}-\frac{1}{r^{2} \sin ^{2} \theta}\right)^{2}\left\langle\psi_{2}\right\rangle .
$$

Applying Eq. (A8) to $\psi_{1}$ and using Eq. (6), we obtain

$$
\Delta \boldsymbol{\psi}_{1}=\boldsymbol{e}_{\varepsilon}\left(\Delta_{r \theta} \psi_{1}-\frac{\psi_{1}}{r^{2} \sin ^{2} \theta}\right) .
$$

With the help of Eq. (A11), the right-hand side of Eq. (A7) is transformed as follows:

$$
\begin{aligned}
\nabla \times & {\left[\left(\Delta \boldsymbol{\psi}_{1}^{*}\right) \times \boldsymbol{v}_{1}\right] } \\
= & \nabla \times\left[\left(\Delta_{r \theta} \psi_{1}^{*}-\frac{\psi_{1}^{*}}{r^{2} \sin ^{2} \theta}\right) \boldsymbol{e}_{\varepsilon} \times\left(v_{1 r} \boldsymbol{e}_{r}+v_{1 \theta} \boldsymbol{e}_{\theta}\right)\right] \\
= & \nabla \times\left[\left(\Delta_{r \theta} \psi_{1}^{*}-\frac{\psi_{1}^{*}}{r^{2} \sin ^{2} \theta}\right)\left(v_{1 r} \boldsymbol{e}_{\theta}-v_{1 \theta} \boldsymbol{e}_{r}\right)\right] \\
= & \frac{\boldsymbol{e}_{\varepsilon}}{r}\left\{\frac{\partial}{\partial r}\left[r v_{1 r}\left(\Delta_{r \theta} \psi_{1}^{*}-\frac{\psi_{1}^{*}}{r^{2} \sin ^{2} \theta}\right)\right]\right. \\
& \left.+\frac{\partial}{\partial \theta}\left[v_{1 \theta}\left(\Delta_{r \theta} \psi_{1}^{*}-\frac{\psi_{1}^{*}}{r^{2} \sin ^{2} \theta}\right)\right]\right\} .
\end{aligned}
$$

Substituting Eqs. (A10) and (A12) into Eq. (A7) leads to Eq. (25).

\section{APPENDIX B: SOLUTION OF EQ. (34)}

For the sake of simplicity, let us denote $x=x_{0}=k_{0} r$. It is also convenient to express the differential operator acting on $\left\langle\psi_{2}^{0 m}\right\rangle$ in Eq. (34) in terms of $x$ and $\mu=\cos \theta$ by

$$
\begin{aligned}
D= & \Delta_{r \theta}-\frac{1}{r^{2} \sin ^{2} \theta}=\frac{k_{0}^{2}}{x^{2}}\left[\frac{\partial}{\partial x}\left(x^{2} \frac{\partial}{\partial x}\right)\right. \\
& \left.+\left(1-\mu^{2}\right) \frac{\partial^{2}}{\partial \mu^{2}}-2 \mu \frac{\partial}{\partial \mu}-\frac{1}{1-\mu^{2}}\right] .
\end{aligned}
$$

As a result, Eq. (34) takes the form:

$$
D^{2}\left\langle\psi_{2}^{0 m}\right\rangle=-\frac{R_{0}}{2 \nu} P_{m}^{1}(\mu) \operatorname{Re}\left\{k_{0}^{5} a_{0}^{*} b_{m} \frac{h_{m}^{(1)}(x)-x h_{m}^{(1) /}(x)}{x^{3}}\right\} .
$$

The right-hand side of Eq. (B2) suggests that a solution can be sought in the following form:

$$
\left\langle\psi_{2}^{0 m}\right\rangle=-\frac{R_{0}}{2 v} P_{m}^{1}(\mu) \operatorname{Re}\left\{k_{0} a_{0}^{*} b_{m} F_{m}(x)\right\},
$$

where $F_{m}(x)$ is a function to be found. Substitution of Eq. (B3) into Eq. (B2) results in

$$
D^{2}\left[P_{m}^{1}(\mu) F_{m}(x)\right]=k_{0}^{4} P_{m}^{1}(\mu) \frac{h_{m}^{(1)}(x)-x h_{m}^{(1) /}(x)}{x^{3}} .
$$


Since $P_{m}^{1}(\mu)$ obeys the equation [20]

$$
\begin{aligned}
& \left(1-\mu^{2}\right) P_{m}^{1 / /}(\mu)-2 \mu P_{m}^{1 /}(\mu)+\left[m(m+1)-\frac{1}{1-\mu^{2}}\right] \\
& \quad \times P_{m}^{1}(\mu)=0
\end{aligned}
$$

the action of the operator $D^{2}$ on the product $P_{m}^{1}(\mu) F_{m}(x)$ results in

$$
\begin{aligned}
D^{2}\left[P_{m}^{1}(\mu) F_{m}(x)\right] \\
=k_{0}^{4} P_{m}^{1}(\mu)\left[\frac{d^{4} F_{m}}{d x^{4}}+\frac{4}{x} \frac{d^{3} F_{m}}{d x^{3}}-\frac{2 m(m+1)}{x^{2}} \frac{d^{2} F_{m}}{d x^{2}}\right. \\
\left.\quad+\frac{m(m+1)\left(m^{2}+m-2\right)}{x^{4}} F_{m}\right]
\end{aligned}
$$

Substitution of Eq. (B6) into Eq. (B4) gives an equation for $F_{m}(x)$,

$$
\begin{aligned}
& \frac{d^{4} F_{m}}{d x^{4}}+\frac{4}{x} \frac{d^{3} F_{m}}{d x^{3}}-\frac{2 m(m+1)}{x^{2}} \frac{d^{2} F_{m}}{d x^{2}} \\
& +\frac{m(m+1)\left(m^{2}+m-2\right)}{x^{4}} F_{m}=G_{m}(x),
\end{aligned}
$$

where

$$
G_{m}(x)=\frac{h_{m}^{(1)}(x)-x h_{m}^{(1) /}(x)}{x^{3}}=\frac{(m+2) h_{m}^{(1)}(x)-x h_{m-1}^{(1)}(x)}{x^{3}} .
$$

Equation (B7) can be solved by the method of variation of parameters, also known as the Lagrange method [27]. According to this method, we first need to find solutions to a homogeneous equation that corresponds to the left-hand side of Eq. (B7),

$$
\begin{gathered}
\frac{d^{4} F_{m}}{d x^{4}}+\frac{4}{x} \frac{d^{3} F_{m}}{d x^{3}}-\frac{2 m(m+1)}{x^{2}} \frac{d^{2} F_{m}}{d x^{2}} \\
+\frac{m(m+1)\left(m^{2}+m-2\right)}{x^{4}} F_{m}=0
\end{gathered}
$$

Partial solutions are sought as $x^{\lambda}$. Substitution of $x^{\lambda}$ into Eq. (B9) leads to a polynomial of fourth order in $\lambda$,

$$
\begin{aligned}
& \lambda(\lambda-1)(\lambda-2)(\lambda+1)-2 m(m+1) \lambda(\lambda-1) \\
& \quad+m(m+1)\left(m^{2}+m-2\right)=0 .
\end{aligned}
$$

The roots of Eq. $(\mathrm{B} 10)$ are $-(m+1),-(m-1), m, m+2$. Therefore, the general solution of Eq. (B9) is written as

$$
F_{m}(x)=\frac{C_{1 m}}{x^{m+1}}+\frac{C_{2 m}}{x^{m-1}}+C_{3 m} x^{m}+C_{4 m} x^{m+2},
$$

where $C_{n m}$ are constants.
To find the solution of Eq. (B7), we set $C_{n m}$ to be functions of $x$,

$$
F_{m}(x)=\frac{C_{1 m}(x)}{x^{m+1}}+\frac{C_{2 m}(x)}{x^{m-1}}+C_{3 m}(x) x^{m}+C_{4 m}(x) x^{m+2} .
$$

According to the method of variation of parameters [27], $C_{n m}(x)$ should obey the following equations:

$$
\begin{aligned}
C_{1 m}^{\prime} y_{1}+C_{2 m}^{\prime} y_{2}+C_{3 m}^{\prime} y_{3}+C_{4 m}^{\prime} y_{4} & =0 \\
C_{1 m}^{\prime} y_{1}^{\prime}+C_{2 m}^{\prime} y_{2}^{/}+C_{3 m}^{\prime} y_{3}^{\prime}+C_{4 m}^{\prime} y_{4}^{\prime} & =0 \\
C_{1 m}^{\prime} y_{1}^{/ /}+C_{2 m}^{\prime} y_{2}^{/ /}+C_{3 m}^{\prime} y_{3}^{/ /}+C_{4 m}^{\prime} y_{4}^{/ /} & =0 \\
C_{1 m}^{\prime} y_{1}^{/ / /}+C_{2 m}^{\prime} y_{2}^{/ / /}+C_{3 m}^{\prime} y_{3}^{/ / /}+C_{4 m}^{/} y_{4}^{/ / /} & =G_{m}(x) .
\end{aligned}
$$

Here, the prime denotes the derivative with respect to $x$ and the functions $y_{n}$ are given by

$$
y_{1}=x^{-(m+1)}, \quad y_{2}=x^{-(m-1)}, \quad y_{3}=x^{m}, \quad y_{4}=x^{m+2} .
$$

Equations (B13) are a system of algebraic equations in the unknowns $C_{n m}^{/}(x)$. We need to calculate these unknowns and then to integrate them over $x$. Doing so, we obtain

$$
C_{1 m}(x)=C_{1 m 0}-\frac{1}{2(2 m+1)(2 m+3)} \int_{\bar{x}_{0}}^{x} G_{m}(s) s^{m+4} d s,
$$

$$
C_{2 m}(x)=C_{2 m 0}+\frac{1}{2(2 m-1)(2 m+1)} \int_{\bar{x}_{0}}^{x} G_{m}(s) s^{m+2} d s,
$$

$$
\begin{aligned}
& C_{3 m}(x)=C_{3 m 0}-\frac{1}{2(2 m-1)(2 m+1)} \int_{\bar{x}_{0}}^{x} G_{m}(s) s^{3-m} d s, \\
& C_{4 m}(x)=C_{4 m 0}+\frac{1}{2(2 m+1)(2 m+3)} \int_{\bar{x}_{0}}^{x} G_{m}(s) s^{1-m} d s,
\end{aligned}
$$

where $\bar{x}_{0}=k_{0} R_{0}$ and $C_{n m 0}$ are constants to be determined by boundary conditions.

To apply the boundary conditions, we first calculate the components of the Eulerian streaming velocity by using Eq. (B3):

$$
\begin{aligned}
\left\langle v_{2 r}^{0 m}\right\rangle & =-\frac{1}{r} \frac{\partial}{\partial \mu}\left(\left\langle\psi_{2}^{0 m}\right\rangle \sqrt{1-\mu^{2}}\right) \\
& =\frac{m(m+1) R_{0}}{2 v r} P_{m}(\mu) \operatorname{Re}\left\{k_{0} a_{0}^{*} b_{m} F_{m}(x)\right\}, \\
\left\langle v_{2 \theta}^{0 m}\right\rangle & =-\frac{1}{r} \frac{\partial}{\partial x}\left(x\left\langle\psi_{2}^{0 m}\right\rangle\right) \\
& =\frac{R_{0}}{2 v r} P_{m}^{1}(\mu) \operatorname{Re}\left\{k_{0} a_{0}^{*} b_{m}\left[F_{m}(x)+x F_{m}^{\prime}(x)\right]\right\} .
\end{aligned}
$$

With the help of the first equation of system $(\mathrm{B} 13), F_{m}^{\prime}(x)$ is calculated from Eq. (B12) as

$$
\begin{aligned}
F_{m}^{\prime}(x)= & -(m+1) \frac{C_{1 m}(x)}{x^{m+2}}-(m-1) \frac{C_{2 m}(x)}{x^{m}} \\
& +m C_{3 m}(x) x^{m-1}+(m+2) C_{4 m}(x) x^{m+1} .
\end{aligned}
$$


The condition of zero streaming velocity at infinity requires that $F_{m}(x) / r \rightarrow 0$ for $r \rightarrow \infty$, which leads to

$$
\begin{gathered}
C_{3 m 0}=\frac{1}{2(2 m-1)(2 m+1)} \int_{\bar{x}_{0}}^{\infty} G_{m}(s) s^{3-m} d s, \\
C_{4 m 0}=-\frac{1}{2(2 m+1)(2 m+3)} \int_{\bar{x}_{0}}^{\infty} G_{m}(s) s^{1-m} d s .
\end{gathered}
$$

In order to calculate $C_{1 m 0}$ and $C_{2 m 0}$, boundary conditions at the bubble surface should be applied. Equations (B19) and (B20) give the components of the Eulerian streaming velocity. To apply the boundary conditions at the bubble surface, we need to know the Lagrangian streaming velocity, which is defined by

$$
\boldsymbol{v}_{L}=\left\langle\boldsymbol{v}_{2}\right\rangle+\boldsymbol{v}_{S},
$$

where $\boldsymbol{v}_{S}$ is the Stokes drift velocity given by [7]

$$
\boldsymbol{v}_{S}=\left\langle\left(\int \boldsymbol{v}_{1} d t \cdot \nabla\right) \boldsymbol{v}_{1}\right\rangle
$$

For modes $n$ and $m$ with $\omega_{n}=\omega_{m}$, Eq. (B25) gives

$$
\boldsymbol{v}_{S}^{n m}=\frac{1}{2 \omega_{n}} \operatorname{Re}\left\{\left[i\left(\boldsymbol{v}_{1 n}+\boldsymbol{v}_{1 m}\right) \cdot \nabla\right]\left(\boldsymbol{v}_{1 n}+\boldsymbol{v}_{1 m}\right)^{*}\right\} .
$$

We need the Stokes drift velocity that is caused by the interaction of modes 0 and $m$. Therefore, we set $n=0$ in Eq. (B26) and keep only cross terms. We also take into account that $v_{10 \theta}=0$ and $\partial v_{10 r} / \partial \theta=0$; see Eqs. (11) and (12). As a result, the components of $\boldsymbol{v}_{S}^{0 m}$ are found from Eq. (B26) to be

$$
\begin{aligned}
& v_{S r}^{0 m}=\frac{1}{2 \omega_{0}} \operatorname{Re}\left\{i v_{10 r} \frac{\partial v_{1 m r}^{*}}{\partial r}+i v_{1 m r} \frac{\partial v_{10 r}^{*}}{\partial r}\right\}, \\
& v_{S \theta}^{0 m}=\frac{1}{2 \omega_{0}} \operatorname{Re}\left\{i v_{10 r} \frac{\partial v_{1 m \theta}^{*}}{\partial r}-\frac{i v_{10 r} v_{1 m \theta}^{*}}{r}\right\} .
\end{aligned}
$$

With the help of Eqs. (11) and (12), one obtains

$$
\begin{gathered}
v_{S r}^{0 m}=\frac{m(m+1) R_{0}}{2 \omega_{0} r^{4}} P_{m}(\mu) \operatorname{Re}\left\{i a_{0}^{*} a_{m}\left(\frac{\bar{x}_{0}}{x}\right)^{m+1}-i a_{0}^{*} b_{m}\left[h_{m}^{(1)}(x)+x h_{m}^{(1) /}(x)\right]\right\}, \\
v_{S \theta}^{0 m}=-\frac{R_{0}}{2 \omega_{0} r^{4}} P_{m}^{1}(\mu) \operatorname{Re}\left\{i(m+3) a_{0}^{*} a_{m}\left(\frac{\bar{x}_{0}}{x}\right)^{m+1}-i a_{0}^{*} b_{m}\left[2 h_{m}^{(1)}(x)-x^{2} h_{m}^{(1) / /}(x)\right]\right\} .
\end{gathered}
$$

From Eqs. (18) and (19) it follows that for $m \geqslant 1$,

$$
a_{m}=\frac{\bar{x}_{m}^{2} h_{m}^{(1) / /}\left(\bar{x}_{m}\right)-(m-1)(m+2) h_{m}^{(1)}\left(\bar{x}_{m}\right)}{2(m+2)} b_{m} .
$$

Substituting Eq. (B31) into Eqs. (B29) and (B30) and keeping in mind that in our case $k_{m}=k_{0}$, one finally obtains

$$
\begin{gathered}
v_{S r}^{0 m}=\frac{m(m+1) R_{0}}{2 \omega_{0} r^{4}} P_{m}(\mu) \operatorname{Re}\left\{i a_{0}^{*} b_{m}\left[\frac{\bar{x}_{0}^{2} h_{m}^{(1) / /}\left(\bar{x}_{0}\right)-(m-1)(m+2) h_{m}^{(1)}\left(\bar{x}_{0}\right)}{2(m+2)}\left(\frac{\bar{x}_{0}}{x}\right)^{m+1}-h_{m}^{(1)}(x)-x h_{m}^{(1) /}(x)\right]\right\}, \\
v_{S \theta}^{0 m}=\frac{R_{0}}{2 \omega_{0} r^{4}} P_{m}^{1}(\mu) \operatorname{Re}\left\{i a_{0}^{*} b_{m}\left[2 h_{m}^{(1)}(x)-x^{2} h_{m}^{(1) / /}(x)-\frac{(m+3)\left[\bar{x}_{0}^{2} h_{m}^{(1) / /}\left(\bar{x}_{0}\right)-(m-1)(m+2) h_{m}^{(1)}\left(\bar{x}_{0}\right)\right]}{2(m+2)}\left(\frac{\bar{x}_{0}}{x}\right)^{m+1}\right]\right\} .
\end{gathered}
$$

Now we can apply the boundary conditions at the bubble surface. Rallabandi et al. [28] have shown that for arbitrary surface periodic deformations, both normal velocity component and tangential stress of the Lagrangian streaming vanish at the mean position of the interface. This means that the following equations are valid:

$$
\begin{gathered}
v_{L r}^{0 m}=\left\langle v_{2 r}^{0 m}\right\rangle+v_{S r}^{0 m}=0 \text { at } r=R_{0}, \\
\frac{1}{r} \frac{\partial v_{L r}^{0 m}}{\partial \theta}+\frac{\partial v_{L \theta}^{0 m}}{\partial r}-\frac{v_{L \theta}^{0 m}}{r}=0 \text { at } r=R_{0} .
\end{gathered}
$$

Substituting Eqs. (B19), (B20), (B32), and (B33) into Eqs. (B34) and (B35), with the help of Eqs. (B12) and (B21), after a cumbersome but straightforward calculation, one obtains

$$
\begin{gathered}
C_{1 m 0}+\bar{x}_{0}^{2} C_{2 m 0}=A_{m}, \\
m(m+2) C_{1 m 0}+\left(m^{2}-1\right) \bar{x}_{0}^{2} C_{2 m 0}=B_{m},
\end{gathered}
$$

where $A_{m}$ and $B_{m}$ are calculated by

$$
\begin{aligned}
& A_{m}=-C_{3 m 0} \bar{x}_{0}^{2 m+1}-C_{4 m 0} \bar{x}_{0}^{2 m+3}-\frac{\bar{x}_{0}^{m-2}}{2}\left[(m+1) h_{m}^{(1)}\left(\bar{x}_{0}\right)+2 \bar{x}_{0} h_{m}^{(1) /}\left(\bar{x}_{0}\right)-\frac{\bar{x}_{0}^{2} h_{m}^{(1) / /}\left(\bar{x}_{0}\right)}{m+2}\right], \\
B_{m}= & \left(1-m^{2}\right) C_{3 m 0} \bar{x}_{0}^{2 m+1}-m(m+2) C_{4 m 0} \bar{x}_{0}^{2 m+3}-\frac{\bar{x}_{0}^{m-2}}{2}\left[\bar{x}_{0}^{3} h_{m}^{(1) / / /}\left(\bar{x}_{0}\right)-\frac{(m+3)(m+5)}{m+2} \bar{x}_{0}^{2} h_{m}^{(1) / /}\left(\bar{x}_{0}\right)\right. \\
& \left.+(m-1)(m+2) \bar{x}_{0} h_{m}^{(1) /}\left(\bar{x}_{0}\right)+(m+1)\left(m^{2}+4 m+1\right) h_{m}^{(1)}\left(\bar{x}_{0}\right)\right] .
\end{aligned}
$$


It follows from Eqs. (B36) and (B37) that

$$
\begin{aligned}
C_{1 m 0} & =\frac{B_{m}-\left(m^{2}-1\right) A_{m}}{2 m+1}, \\
C_{2 m 0} & =\frac{m(m+2) A_{m}-B_{m}}{(2 m+1) \bar{x}_{0}^{2}} .
\end{aligned}
$$

To sum up, we have shown that the solution of Eq. (34) is given by Eq. (B3), which in turn leads to Eqs. (B19) and (B20), which give the Eulerian streaming velocity. We have calculated all the quantities which appear in the above equations. In the course of this calculation, we have also calculated the Stokes drift velocity, which, when being added to the Eulerian streaming velocity, gives the Lagrangian streaming velocity.

[1] Ultrasound, Its Chemical, Physical, and Biological Effects, edited by K. S. Suslick (VCH, Verlagsgesellschaft, Berlin, 1988).

[2] W. L. Nyborg, Acoustic streaming, in Physical Acoustics, edited by W. P. Mason (Academic, New York, 1965), Vol. IIB, pp. 266-331.

[3] J. Kolb and W. L. Nyborg, Small-scale acoustic streaming in liquids, J. Acoust. Soc. Am. 28, 1237 (1956).

[4] S. A. Elder, Cavitation microstreaming, J. Acoust. Soc. Am. 31, 54 (1959).

[5] B. J. Davidson and N. Riley, Cavitation microstreaming, J. Sound Vib. 15, 217 (1971).

[6] J. Wu and G. Du, Streaming generated by a bubble in an ultrasound field, J. Acoust. Soc. Am. 101, 1899 (1997).

[7] M. S. Longuet-Higgins, Viscous streaming from an oscillating spherical bubble, Proc. R. Soc. London, Ser. A 454, 725 (1998).

[8] A. O. Maksimov, Viscous streaming from surface waves on the wall of acoustically-driven gas bubbles, Eur. J. Mech. B. Fluids 26, 28 (2007).

[9] A. A. Doinikov and A. Bouakaz, Acoustic microstreaming around a gas bubble, J. Acoust. Soc. Am. 127, 703 (2010).

[10] T. A. Spelman and E. Lauga, Arbitrary axisymmetric steady streaming: Flow, force and propulsion, J. Eng. Math. 105, 31 (2017).

[11] T. G. Leighton, From seas to surgeries, from babbling brooks to baby scans: The acoustics of gas bubbles in liquids, Int. J. Modern Phys. B 18, 3267 (2004).

[12] P. Marmottant, J. Raven, H. Gardeniers, J. Bomer, and S. Hilgenfeldt, Microfluidics with ultrasound-driven bubbles, J. Fluid Mech. 568, 109 (2006).

[13] D. Gritsenko, Y. Lin, V. Hovorka, Z. Zhang, A. Ahmadianyazdi, and $\mathrm{J} . \mathrm{Xu}$, Vibrational modes prediction for water-air bubbles trapped in circular microcavities, Phys. Fluids 30, 082001 (2018).

[14] M. Guédra, C. Inserra, C. Mauger, and B. Gilles, Experimental evidence of nonlinear mode coupling between spherical and nonspherical oscillations of microbubbles, Phys. Rev. E 94, 053115 (2016).

[15] M. Guédra, C. Inserra, B. Gilles, and C. Mauger, Periodic onset of bubble shape instabilities and their influence on the spherical mode, in Proceedings of the IEEE International Ultrasonics
Symposium (IUS), Tours, France (IEEE, New York, 2016), pp. $1-4$.

[16] M. Guédra, S. Cleve, C. Mauger, P. Blanc-Benon, and C. Inserra, Dynamics of nonspherical microbubble oscillations above instability threshold, Phys. Rev. E 96, 063104 (2017).

[17] M. S. Plesset, On the stability of fluid flows with spherical symmetry, J. Appl. Phys. 25, 96 (1954).

[18] L. D. Landau and E. M. Lifshitz, Fluid Mechanics (Pergamon Press, Oxford, 1987).

[19] G. Arfken, Mathematical Methods for Physicists (Academic Press, San Diego, CA, 1985).

[20] M. Abramowitz and I. N. Stegun, Handbook of Mathematical Functions (Dover Publications, New York, 1965).

[21] A. A. Doinikov and A. Bouakaz, Effect of a distant rigid wall on microstreaming generated by an acoustically driven gas bubble, J. Fluid Mech. 742, 425 (2014).

[22] P. J. Westervelt, The theory of steady rotational flow generated by a sound field, J. Acoust. Soc. Am. 25, 60 (1953).

[23] See Supplemental Material at http://link.aps.org/supplemental/ 10.1103/PhysRevE.100.033104 for a MATLAB code that implements the calculation of the Langragian streaming velocity for the 0 -m case (MainProgram_Case_0m.m).

[24] S. Cleve, M. Guédra, C. Mauger, C. Inserra, and P. BlancBenon, Experimental investigation of microstreaming induced by free nonspherically oscillating microbubbles, Proc. Mtgs. Acoust. 34, 045030 (2018).

[25] S. Cleve, M. Guédra, C. Mauger, C. Inserra, and P. BlancBenon, Microstreaming around acoustically trapped, nonspherically oscillating microbubbles, J. Fluid. Mech. 875, 597 (2019).

[26] S. Cleve, M. Guédra, C. Inserra, C. Mauger, and P. BlancBenon, Surface modes with controlled axisymmetry triggered by bubble coalescence in a high-amplitude acoustic field, Phys. Rev. E. 98, 033115 (2018).

[27] W. E. Boyce and R. C. DiPrima, Elementary Differential Equations and Boundary Value Problems (Wiley, New York, 2001).

[28] B. Rallabandi, C. Wang, and S. Hilgenfeldt, Two-dimensional streaming flows driven by sessile semicylindrical microbubbles, J. Fluid Mech. 739, 57 (2014). 